\title{
Effects of intraocular miotics on cultured bovine corneal endothelium
}

\author{
JEFFREY L. JAY AND MARGARET MACDONALD \\ From the Tennent Institute of Ophthalmology, University of Glasgow, Glasgow
}

SUMMARY Two cases of severe corneal oedema occurred after the use of intraocular pilocarpine. Experimental investigations were conducted with cultured bovine corneal endothelial cells exposed for 5 minutes to $1 \%$ pilocarpine solutions of varying composition. Cells were destroyed in solutions not isotonic with aqueous humour, and calcium-free ionic solutions caused loss of cell adhesion without loss of viability. Low $\mathrm{pH}$ or the presence of $1 \%$ pilocarpine had no detectable effects; $1 \%$ acetylcholine chloride in 5\% mannitol (Miochol) also caused cell destruction, and this preparation was found to be considerably hypertonic. The minimum requirements for the formulation of intraocular miotics are discussed.

Corneal oedema after operation for cataract is usually an iatrogenic disorder caused by mechanical and chemical injury to the endothelium of the cornea. This investigation was carried out after 2 cases of severe corneal oedema had followed the accidental use of an inappropriate intraocular miotic. The preparation used was a sterile, preservative-free solution of $1 \%$ pilocarpine nitrate in water in a single-dose container (Minims, Smith \& Nephew Pharmaceuticals Ltd.). This miotic has a $\mathrm{pH}$ of 4.5 and an osmolality of approximately $78 \mathrm{mOs} /$ litre and is not designed for intraocular use.

Experiments were set up to study the morphological changes induced in bovine corneal endothelial cell culutures in vitro after a brief exposure to various miotic preparations. The relative toxic effects of $\mathrm{pH}$, osmolality, absence of calcium, or presence of pilocarpine were investigated. The probable cause of the corneal oedema noted in the 2 clinical cases included in this report was identified, and the minimum requirements for the formulation of a miotic solution for intraocular use are outlined.

\section{Case reports}

CASE 1

An apparently uncomplicated intracapsular cryoextraction for presenile nuclear cataract was performed on a 51-year-old woman. Preoperative examination of the cornea by specular reflection with the slit-lamp microscope had shown slight

Address for reprints: Dr J. L. Jay, Tennent Institute of Ophthalmology, 38 Church Street, Glasgow G11 6NT

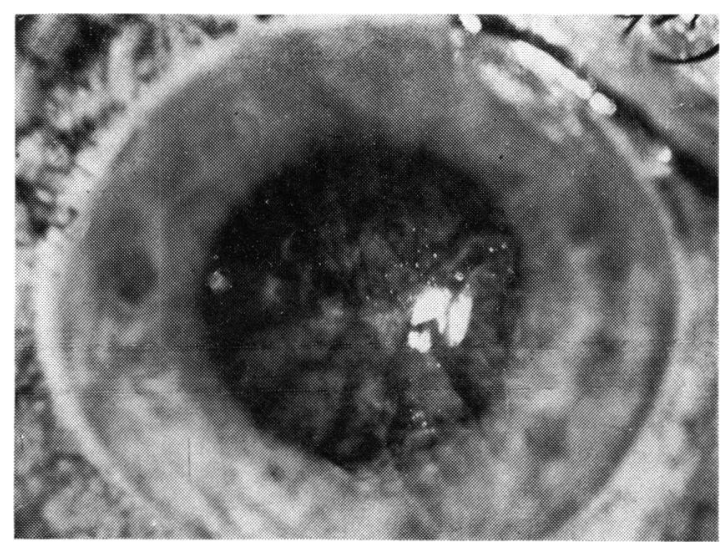

Fig. 1 Severe corneal oedema 24 hours after intracapsular lens extraction; $1 \%$ pilocarpine nitrate in water had been used as an intraocular miotic (Case l)

cornea guttata but an otherwise healthy endothelial mosaic. At the end of the operation the anterior chamber was reformed with a sterile solution of $1 \%$ pilocarpine nitrate in water without preservative (Minims). On the first postoperative day severe corneal oedema was present (Fig. 1) and only a $1-\mathrm{mm}$ peripheral band of the inferior part of the cornea remained clear. The eye healed quickly without further complication, but after 7 months there had been no improvement in the bullous keratopathy.

CASE 2

A 69-year-old woman had a similar procedure performed by the same surgeon on the same operating list. Again preoperative assessment of the 
Table 1 Miotic solutions and their effect on bovine corneal endothelial cell cultures

\begin{tabular}{|c|c|c|c|c|c|}
\hline \multirow{2}{*}{ Solution } & \multirow{2}{*}{$p H$} & \multirow{2}{*}{$\begin{array}{l}\text { Osmolality } \\
(\mathrm{mOs} / \text { litre })\end{array}$} & \multirow{2}{*}{$\begin{array}{l}\text { Number of } \\
\text { experiments }\end{array}$} & \multicolumn{2}{|l|}{ Effect } \\
\hline & & & & Cytotoxic & Rounding \\
\hline $1 \%$ pilocarpine in water & $4 \cdot 5$ & 81 & 5 & 4 & 0 \\
\hline $1 \%$ pilocarpine isotonic in $\mathrm{NaCl}$ & $4 \cdot 6$ & 282 & 5 & 0 & 3 \\
\hline $1 \%$ pilocarpine isotonic in $\mathrm{NaCl}$ with $\mathrm{CaCl}_{2} *$ & $4 \cdot 6$ & 308 & 3 & 0 & 0 \\
\hline $1 \%$ pilocarpine isotonic in PBS + & $7 \cdot 2$ & 300 & 5 & $\mathbf{0}$ & 4 \\
\hline Isotonic PBS & $7 \cdot 9$ & 296 & 4 & 0 & 2 \\
\hline $1 \%$ acetylcholine in $5 \%$ mannitol + & $6 \cdot 0$ & 430 & 8 & 4 & 0 \\
\hline $5 \%$ mannitol & 7.5 & 292 & 4 & 0 & 0 \\
\hline
\end{tabular}

*Calcium chloride $200 \mathrm{mg} / \mathrm{l}$ (anhydrous). †Phosphate buffered saline $\left(\mathrm{NaH}_{2} \mathrm{PO}_{4}, 2 \mathrm{H}_{2} \mathrm{O} / \mathrm{Na}_{2} \mathrm{HPO}_{4}, 12 \mathrm{H}_{2} \mathrm{O}\right)$. $\ddagger$ Miochol (Cooper Laboratories)

corneal endothelium showed mild guttate change on the endothelial surface but no gross disturbance of the endothelial cell pattern. The anterior chamber was reformed with an identical solution of pilocarpine. Severe oedema of the whole cornea was seen on the first postoperative day, but during the following 3 months the oedema cleared slowly from below. Observation of the corneal endothelium after the oedema had cleared showed severe guttate changes with a distorted cell mosaic. The unoperated eye showed a normal endothelial cell pattern.

\section{Material and methods}

Adult bovine corneas were removed from eyes obtained at the local slaughterhouse. Within 4 hours of death the endothelial cell layer together with Descemet's membrane was separated by dissection as described by Stocker et al. (1958). The membrane was cut into small pieces with scissors and transferred to plastic tissue culture flasks of $25 \mathrm{~cm}^{2}$ surface area. Hepes buffered TC 199 culture medium was used with $10 \%$ fetal calf serum and benzyl penicillin 100 units $/ \mathrm{ml}$. The cultures were maintained at $37^{\circ} \mathrm{C}$. Secondary cultures were obtained by trypsinisation of primary explants with $5 \%$ trypsin in calcium- and magnesium- free phosphate buffered saline (Dulbecco's). Tests were performed on established cell cultures which had been maintained for more than 1 year and also on fresh cultures only a few weeks old.

To imitate the time scale of a single dose of intraocular miotic experiments were designed to study the endothelial cell changes after a 5-minute exposure to various test solutions and during 24 hours of recovery in culture medium. $2 \mathrm{ml}$ of test or control solutions (Table 1) were exchanged for the culture medium in each flask and allowed to remain in contact with the cell monolayer for 5 minutes. The solution was poured off and the cells washed with $2 \mathrm{ml}$ of TC 199. The cells were then allowed to recover in the growth medium described above. Four fields in each flask were marked for photography and a series of phase-contrast photomicrographs were taken of each field. The time intervals were: before exposure to the solution, after 5 minutes' exposure, after 2 hours' recovery in growth medium, and after 24 hours' recovery. The entire experiment was carried out in a hot room at $37^{\circ} \mathrm{C}$, and during each experiment several different test solutions were compared with control preparations.

The photomicrographs were printed at $\times 300$ magnification and with the identity of the test solution masked; changes in the appearance of the cells in each sequence were recorded. When the analysis of the photographs was complete, the solutions were identified.

Test solutions were made up as shown in Table 1 so that possible toxic effects of osmolality, $\mathrm{pH}$, absence of calcium ions, or presence of pilocarpine could be differentiated. Osmolality was determined by depression of freezing point using a model 3D 'Advance' osmometer, and $\mathrm{pH}$ was measured on a blood gas analyser. For the purposes of comparison the commercial preparation of $1 \%$ acetylcholine chloride in $5 \%$ mannitol (Miochol, Cooper Laboratories) was also tested, as was an isotonic solution of $5 \%$ mannitol. A pilot experiment included sterile distilled water as one of the test fluids.

\section{Results}

MORPHOLOGICAL CHANGES IN CULTURED ENDOTHELIUM

Two distinct changes in cell morphology were noted in the cultures. Some solutions produced cell destruction which was not obvious at the end of the 


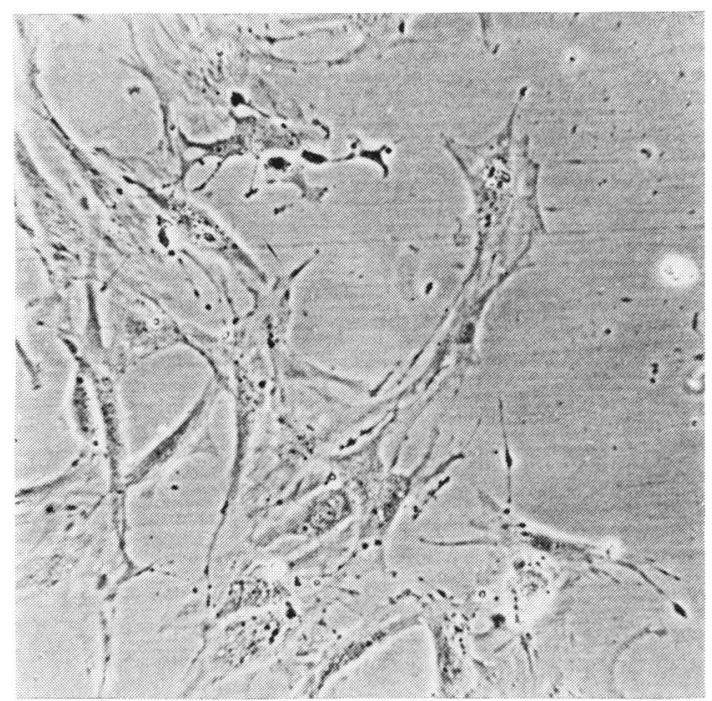

$2 a$

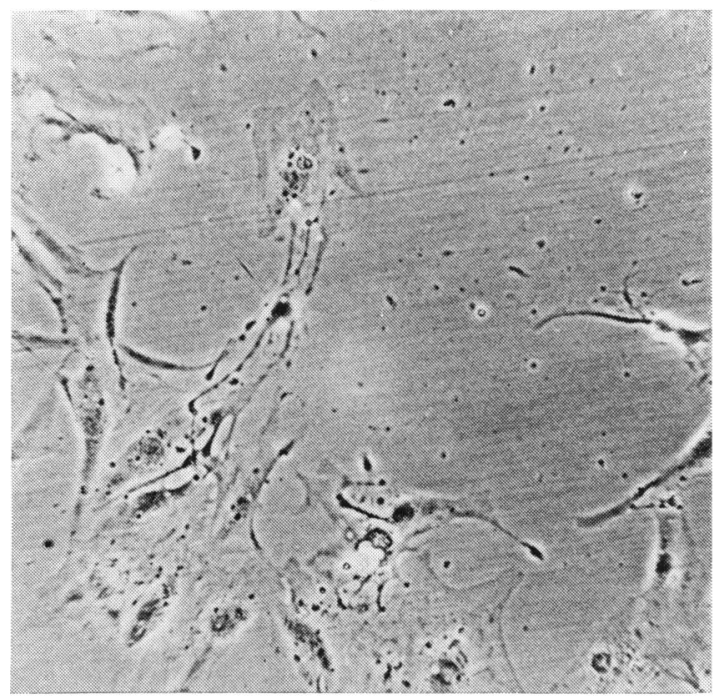

$2 c$

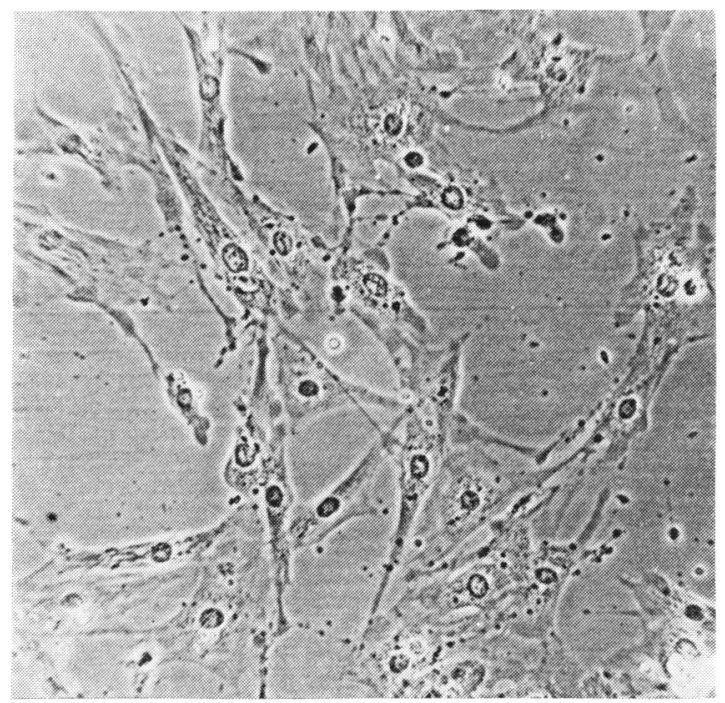

$2 b$

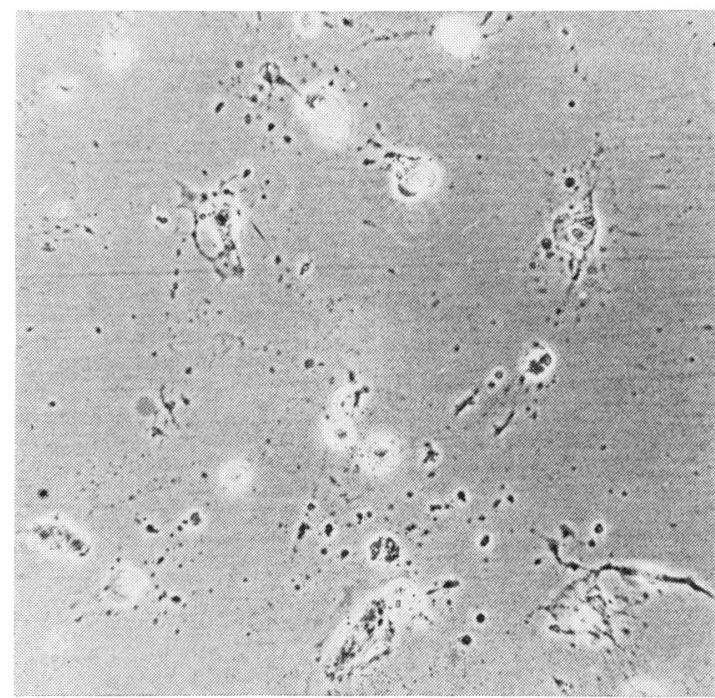

$2 d$

Fig. 2 Morphological changes in subcultured bovine corneal endothelial cells induced by exposure to $1 \%$ pilocarpine nitrate in water; pH 4.5, $81 \mathrm{mOs} / \mathrm{l}$, for 5 minutes. (a) Before exposure, the polygonal cell forms are typical of actively spreading endothelium. (b) The same cells after 5 minutes' exposure; the nuclei are much more prominent and a perinuclear halo is seen. (c) After 2 hours' recovery in TC 199 culture medium the cells show some cytoplasmic disruption. (d) At 24 hours severe cell destruction has occurred (phase contrast, $\times 100$ )

5 minutes' exposure but developed during the subsequent 24 hours in normal growth medium. This is called the cytotoxic effect (Fig. 2). Other test solutions caused the cells to lose adhesion and separate from each other and from the substrate. This is described as cell rounding, and it occurred as soon as the solutions were added to the culture flasks.
Residual cells rapidly regained their normal morphology during the recovery phase (Fig. 3).

These changes were seen both in actively dividing areas at the edges of cell colonies and in confluent areas at the centre of the colonies, but the active cell forms showed more marked changes of either type. Recently established cultures seemed just as 


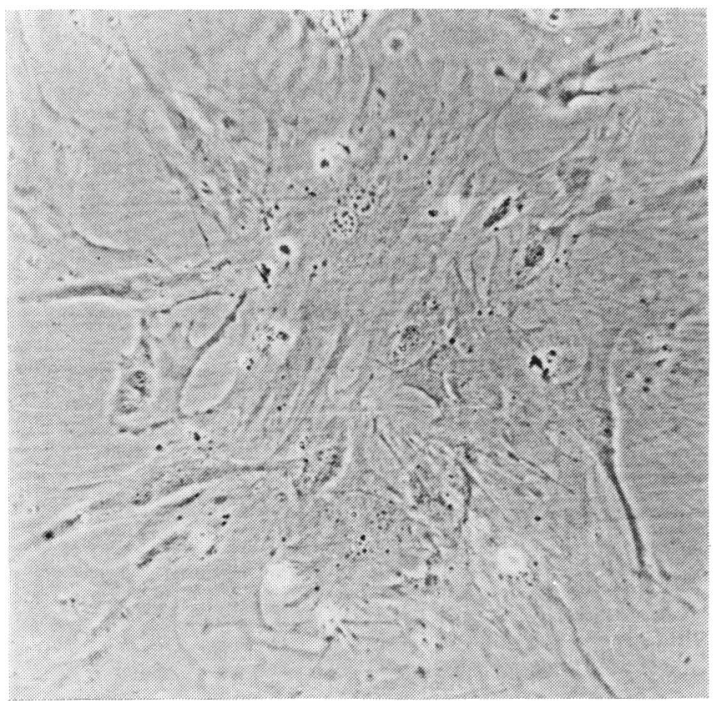

$3 a$

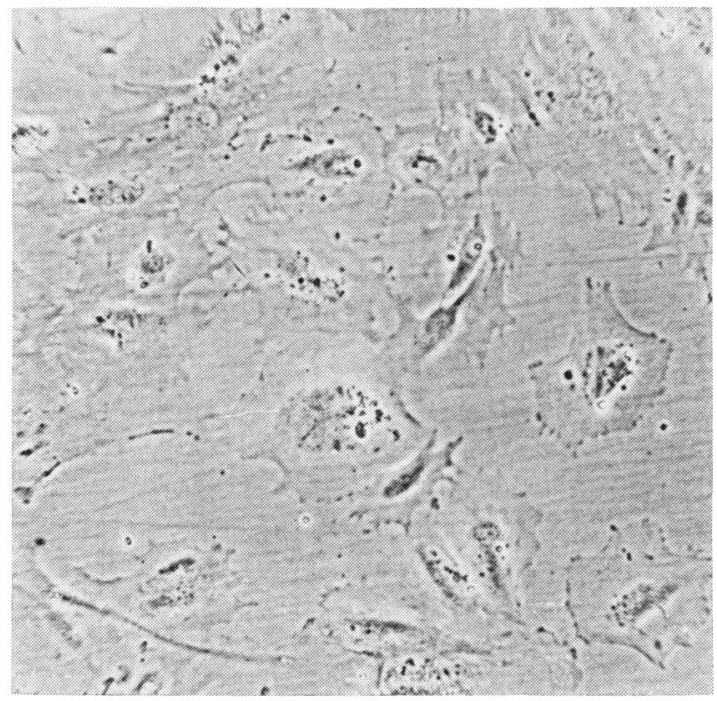

$3 c$

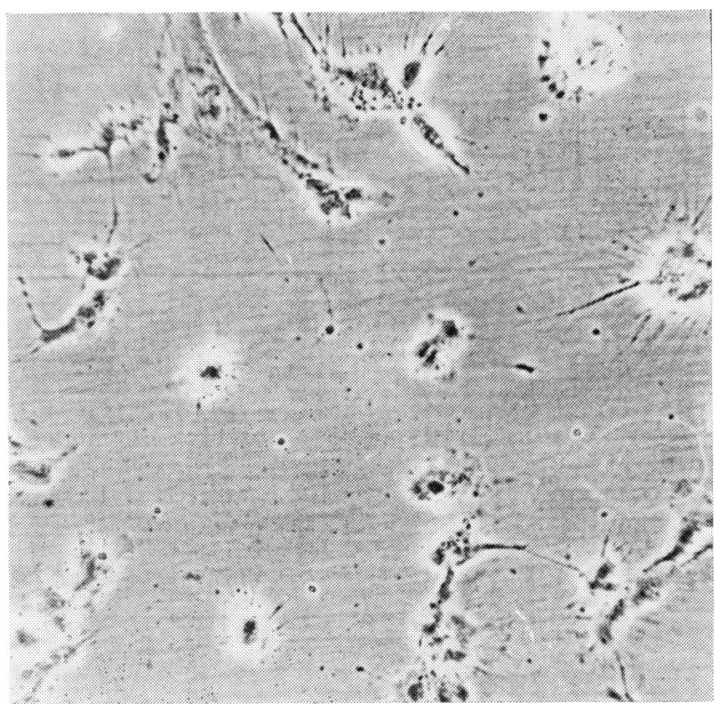

$3 b$

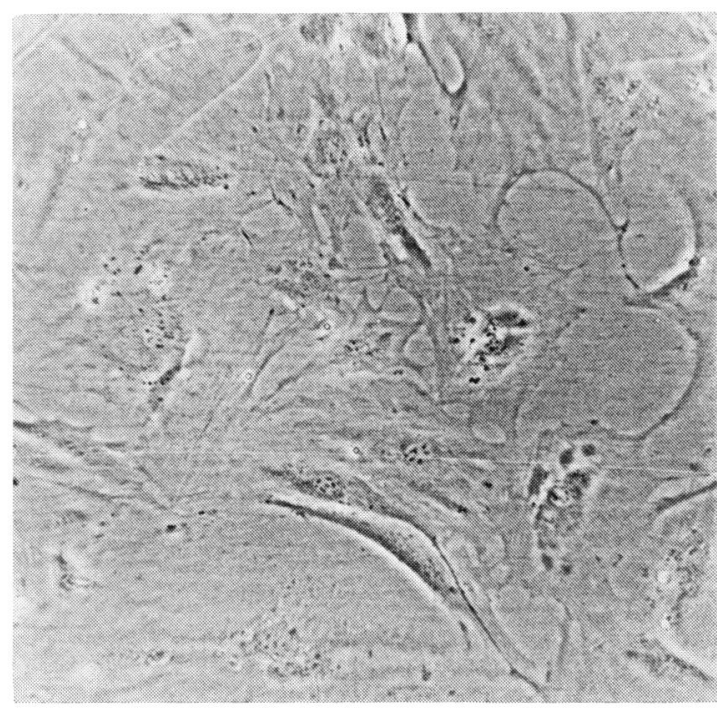

$3 d$

Fig. 3 Response of subcultured bovine endothelial cells to calcium-free ionic solutions. (a) Initial appearance. (b) The same area after 5 minutes' exposure to $1 \%$ pilocarpine nitrate isotonic in sodium chloride. Cells lose adhesion to the substrate and become rounded. (c) After 2 hours' recovery in TC 199 culture medium the cells have again fattened and spread out. (d) After 24 hours' recovery (phase contrast, $\times 100$ )

susceptible to the test solution as did cultures which had been established for over a year.

\section{EFFECTS OF SOLUTIONS UNDER}

INVESTIGATION

Cytotoxic effect.-After 5 minutes' exposure to $1 \%$ pilocarpine nitrate in aqueous solution $(\mathrm{pH} \mathrm{4.5,}$
$81 \mathrm{mOs} /$ litre) the cells showed increased prominence of nuclei with a clear perinuclear halo (Fig. 2b). These cells showed some distruption of cytoplasm in the early recovery period at 2 hours (Fig. $2 c$ ), and by 24 hours (Fig. $2 d$ ) appreciable cell death had occurred. Similar but more severe changes occurred when the cells were exposed to distilled water. 
$1 \%$ acetylcholine chloride in $5 \%$ mannitol (Miochol; pH $60,430 \mathrm{mOs} /$ litre) also caused appreciable cell destruction after 24 hours' recovery, although there were no detectable changes in the ealier photographs. Bo:h these preparations causing cell damage had stibjected the cells to considerable osmotic shock.

Rounding.-Exposure to isotonic calcium-free ionic solutions (Table 1) caused the cells to separate, lose adhesion to the substrate, and form spheres which were visible in the 5-minute micrographs (Fig. 3b). This did not affect the viability of the cells, as rounded cells still in contact with the substrate rapidly flattened on to the bottom of the flasks during the recovery period (Fig. $3 c$ and $3 d$ ). Again actively spreading cells showed greater changes than did confluent cell areas at the centre of colonies.

Table 1 shows that the cytotoxic effects occurred only in non-isotonic solutions while rounding occurred only in isotonic calcium-free ionic solutions. The experiment did not show any identifiable changes which could be attributed to the low pH of some solutions or to the presence of pilocarpine.

$1 \%$ pilocarpine isotonic in sodium chloride with $200 \mathrm{mg}$ of calcium chloride per litre, $\mathrm{pH} 4.5$, had no detectable effect on the cells after 5 minutes' exposure. An isotonic solution of $5 \%$ mannitol was also without effect.

\section{Discussion}

The experimental results suggest that the severe corneal oedema which occurred after the 2 cases of intracapsular lens extraction was related to the extreme osmotic shock which was caused by the intraocular use of a solution of $1 \%$ pilocarpine nitrate in water. The presence of pilocarpine when isotonic in saline with added calcium had no detectable effect on bovine endothelial cell cultures after 5 minutes' exposure, but after several hours' exposure to pilocarpine a dose-related toxic effect does occur (Coles, 1975a).

Addition of a phosphate buffer to neutralise the rather acid pilocarpine solutions showed no detectable benefit and may have accelerated the rounding effect by combining with residual calcium in the cells to enhance the effect of a calcium-free solution. Buffered preparations would have the disadvantage of rendering the miotic unstable, as pilocarpine in neutral solution undergoes spontaneous isomerisation to inactive isopilocarpine after a few weeks of storage at room temperature (Anderson and Cowle, 1968). The theoretical benefit of using a neutral pilocarpine for intraocular injection would have to be balanced by the risk of cold shock to the corneal endothelium if the buffered pilocarpine required storage at low temperature.
Although it has been shown by Kaye et al. (1968) that calcium is essential to maintain the junctional complexes of endothelial cells, it is unlikely that the omission of this divalent cation would cause appreciable endothelial cell death. On the other hand the cell rounding and loss of adhesion demonstrated in this study might be a factor in causing temporary disruption of the continuity of the endothelial barrier in vivo. With more severe loss of adhesion some cells might separate completely from Descemet's membrane, so that even after recovery there would be a reduction in the endothelial cell population. This might be enough to provoke decompensation in a cornea with an already reduced endothelial cell density.

The damage produced by $1 \%$ acetylcholine in $5 \%$ mannitol may be related to the hypertonicity of this preparation, but this has not yet been fully clarified. Mannitol alone in isotonic strength $(5 \%)$ did not seem to cause changes.

Extracapsular lens extraction, phacoemulsifcation, intraocular lens implantation, and the use of suction cutting instruments in the anterior segment may require prolonged irrigation of the anterior chamber with an artificial aqueous humour. Perfusion fluids should not be toxic to the corneal endothelium, as the use of an unphysiological solution is likely to potentiate any mechanical disruption of the endothelium during the surgical manipulation. A suitable artificial aqueous for prolonged perfusion has been shown by Edelhauser et al. (1976) to require a bicarbonated Ringer's solution with added glucose, glutathione, and adenosine (GBR). The same authors suggest that for low volume irrigation of the anterior chamber less favourable fluids such as sodium chloride $0.9 \%$ or Ringer-lactate solution might be adequate.

As miotics for intraocular use are likely to bathe the corneal endothelium only for a short time, their formulation need be less complex than that for solutions used for anterior chamber perfusion. There are, however, some important factors to be observed. The pilocarpine should be prepared in a physiological salt solution containing calcium to maintain cell adhesion, and the final osmolality must be near that of aqueous humour, approximately $300 \mathrm{mOs} /$ litre. No bacteriostatic preservative should be used, as these may cause severe endothelial damage. Maurice and Perlman (1977) have shown that benzalkonium chloride in the concentration used in eye drops causes irreversible destruction of rabbit corneal endothelial function after a few seconds of exposure. In addition toxic effects of intraocular epinephrine have been attributed by Hull et al. (1975) to the bisulphate preservative rather than the drug itself, and Coles (1975b) has shown 
that the endothelial damage caused by erythromycin is aggravated by the addition of benzyl alcohol preservative.

Autoclave sterilisation is suitable for pilocarpine and may be preferable to filter sterilisation, as some membrane filters are coated with a detergent wetting agent which can damage cell cultures if the filters are not washed thoroughly before use. Alternatively, grades of filters specifically for tissue culture media may be used, as these have the wetting agent omitted (Mulvany, 1972).

\section{References}

Anderson, R. A., and Cowle, J. B. (1968). Influence of pH on the effect of pilocarpine on aqueous dynamics. British Journal of Ophthalmology, 52, 607-611.

Coles, W. H. (1975a). Pilocarpine toxicity. Archives of Ophthalmology, 93, 36-41.

Coles, W. H. (1975b). Effects of antibiotics on the in vitro rabbit corneal endothelium. Investigative Ophthalmology, 14, 246-250.

Edelhauser, H. F., van Horn, D. L., Schultz, R. O., and Hyndiuk, R. A. (1976). Comparative toxicity of intraocular irrigating solutions on the corneal endothelium. American Journal of Ophthalmology, 81, 473-481.

Hull, D. S., Chemotti, M. T., Edelhauser, H. F., van Horn, D. L., and Hyndiuk, R. A. (1975). Effect of epinephrine on the corneal endothelium. American Journal of Ophthalmology, 79, 245-250.

Kaye, G. I., Mishima, S., Cole, J. D., and Kaye, N. W. (1968). Studies on the cornea. VII. Effects of perfusion with a calcium-free medium on the corneal endothelium. Investigative Ophthalmology, 7, 53-66.

Maurice, D., and Perlman, M. (1977). Permanent destruction of the corneal endothelium in rabbits. Investigative Ophthalmology, 16, 646-649.

Mulvany, J. G. (1972). Sterile filtration. In Animal Tissue Culture: Advances in Technique, p. 27. Edited by G. D. Walsey. Butterworths: London.

Stocker, F. W., Eiring, A., Giorgiade, R., and Giorgiade, N. (1958). A tissue culture technique for growing corneal epithelial, stromal, and endothelial tissues separately. American Journal of Ophthalmology, 46, 294-298. 